\title{
Bulky iminophosphonamines for N-P-N coordination: Synthesis and structural characterization of lithium iminophosphonamides and homoleptic bis-chelates of $\mathrm{Co}(\mathrm{II}), \mathrm{Ni}$ (II) and $\mathrm{Cu}$ (II)
}

\author{
BILLA PRASHANTH and SANJAY SINGH* \\ Department of Chemical Sciences, Indian Institute of Science Education and Research Mohali, \\ Knowledge City, Sector 81, SAS Nagar, Mohali 140 306, India \\ e-mail: sanjaysingh@iisermohali.ac.in
}

MS received 19 September 2013; accepted 21 July 2014

\begin{abstract}
Two new sterically demanding iminophosphonamine ligands $\left(2,4,6-\mathrm{Me}_{3} \mathrm{C}_{6} \mathrm{H}_{2} \mathrm{NH}\right) \mathrm{P}(\mathrm{Ph} 2)=\mathrm{N}$ $\left(\mathrm{C}_{6} \mathrm{H}_{2}-2,4,6-\mathrm{Me}_{3}\right)$ (1) and $\left(2,6-i \mathrm{Pr}_{2} \mathrm{C}_{6} \mathrm{H}_{3} \mathrm{NH}\right) \mathrm{P}\left(\mathrm{Ph}_{2}\right)=\mathrm{N}\left(\mathrm{C}_{6} \mathrm{H}_{2}-2,4,6-\mathrm{Me}_{3}\right)$ (2) and their lithium derivatives as tmeda adducts (Li·tmeda) $\left[\left(2,4,6-\mathrm{Me}_{3} \mathrm{C}_{6} \mathrm{H}_{2}\right) \mathrm{NP}\left(\mathrm{Ph}_{2}\right)=\mathrm{N}\left(\mathrm{C}_{6} \mathrm{H}_{2}-2,4,6-\mathrm{Me}_{3}\right)\right]$ (3) and (Li.tmeda) [(2,6$\left.\left.i \mathrm{Pr}_{2} \mathrm{C}_{6} \mathrm{H}_{3}\right) \mathrm{NP}\left(\mathrm{Ph}_{2}\right)=\mathrm{N}\left(\mathrm{C}_{6} \mathrm{H}_{2}-2,4,6-\mathrm{Me}_{3}\right)\right](4)$, respectively are reported here. Compounds $\mathbf{1}-\mathbf{4}$ have been investigated by ${ }^{1} \mathrm{H},{ }^{13} \mathrm{C}$ and ${ }^{13} \mathrm{P}\left\{{ }^{1} \mathrm{H}\right\}$ NMR spectroscopy. The ${ }^{7} \mathrm{Li}$ NMR for complexes $\mathbf{3}$ and $\mathbf{4}$ has also been reported. Utility of the ligands and their lithium derivatives have been shown in the synthesis of bis-homoleptic metal complexes $\mathrm{M}\left[\mathrm{Ph}_{2} \mathrm{P}\left(\mathrm{NC}_{6} \mathrm{H}_{2}-2,4,6-\mathrm{Me}_{3}\right)_{2}\right]_{2}(\mathrm{M}=\mathrm{Co}(5), \mathrm{Cu}$ (6) and $\mathrm{Ni}$ (7). Metal-bis-silylamide generated in situ was reacted with the ligand (for $\mathbf{5}$ and $\mathbf{6}$ ) or the lithium derivative of the ligand was reacted with the metal chloride (for 7). Molecular structure of compounds 1-7 has been elucidated by single crystal X-ray diffraction analyses. The complexes are formed in good yields and are highly lipophilic in a wide range of solvents.
\end{abstract}

Keywords. Metallacycles; monoanion; homoleptic complexes; iminophosphonamides.

\section{Introduction}

The NXN ligands have commonly been employed in the synthesis of four-membered metallacycles containing main group, transition as well as inner transition elements $\left(\mathrm{X}=\mathrm{CR}, \mathrm{N}, \mathrm{SR}, \mathrm{PR}_{2}\right) .{ }^{1-16}$ Among this class of ligands, the iminophosphonamides offer the longest $\mathrm{X}$ $\mathrm{N}$ bond length $(1.60 \AA)$, which in turn allows a wider $\mathrm{N}-$ $\mathrm{M}-\mathrm{N}$ bite angle (usually $70-76^{\circ}$ ). The sulphinamidinate resembles $\left(1.60-1.62 \AA\right.$ and $\left.70-75^{\circ}\right)$ the iminophosphonamides closely but the former has gained attention recently. ${ }^{10-12}$ Another important feature of iminophosphonamides is that most of the $\mathrm{N}_{2} \mathrm{PM}$ metallacycles are nearly planar. The N-P-N coordination of iminophosphonamides has been realized in the form of homoleptic and heteroleptic four-membered chelates. The range of metal ions spans over $s, p, d$ blocks and recently a few lanthanide complexes were also reported. ${ }^{17-32}$ Some of these metal complexes are useful as catalysts and promise a wide scope for future research. ${ }^{26-32}$

In our ongoing research interest to fine tune the steric and electronic factors of the ligands ${ }^{33}$, we have attempted to synthesize sterically bulky iminophosphonamides and to use them in the synthesis

*For correspondence of metallacycles. Consequently, we report here the synthesis and characterization of two new iminophosphonamine ligands $\left(2,4,6-\mathrm{Me}_{3} \mathrm{C}_{6} \mathrm{H}_{2} \mathrm{NH}\right) \mathrm{P}\left(\mathrm{Ph}_{2}\right)=\mathrm{N}$ $\left(\mathrm{C}_{6} \mathrm{H}_{2}-2,4,6-\mathrm{Me}_{3}\right)(\mathbf{1})$ and $\left(2,6-i \mathrm{Pr}_{2} \mathrm{C}_{6} \mathrm{H}_{3} \mathrm{NH}\right) \mathrm{P}\left(\mathrm{Ph}_{2}\right)=\mathrm{N}$ $\left(\mathrm{C}_{6} \mathrm{H}_{2}-2,4,6-\mathrm{Me}_{3}\right)(2)$ and their corresponding lithium derivatives as tmeda adducts (Li·tmeda) $\left[\left(2,4,6-\mathrm{Me}_{3} \mathrm{C}_{6}\right.\right.$ $\left.\left.\mathrm{H}_{2}\right) \mathrm{NP}\left(\mathrm{Ph}_{2}\right)=\mathrm{N}\left(\mathrm{C}_{6} \mathrm{H}_{2}-2,4,6-\mathrm{Me}_{3}\right)\right](3)$ and (Li.tmeda) $\left[\left(2,6-i \mathrm{Pr}_{2} \mathrm{C}_{6} \mathrm{H}_{3}\right) \mathrm{NP}\left(\mathrm{Ph}_{2}\right)=\mathrm{N}\left(\mathrm{C}_{6} \mathrm{H}_{2}-2,4,6-\mathrm{Me}_{3}\right)\right]$ (4). Application of the ligands and their lithium derivatives are shown in the synthesis of bis-homoleptic metal complexes of $\mathrm{Co}(\mathrm{II})(5), \mathrm{Cu}(\mathrm{II})$ (6) and $\mathrm{Ni}(\mathrm{II})$ (7). All these complexes have shown good lipophilicity in a wide range of solvents and have been isolated in good yields.

Iminophosphonamides of lithium reported in the literature comprise of trans-1,2-diaminocyclohexanebridged bis-iminophosphonamide, ${ }^{21}\left[\left\{\mathrm{R}_{2} \mathrm{P}\left(\mathrm{NSiMe}_{3}\right)_{2}\right\}\right.$ $\left.\mathrm{Li}(\mathrm{X})_{2}\right] \quad(\mathrm{R}=t \mathrm{Bu}$ or $\mathrm{Ph}$ and $\mathrm{X}=\mathrm{THF}$ or $\mathrm{Py})^{17-19}$ and $\left(\mathrm{Ph}_{2} \mathrm{P}-\mathrm{N}\right)_{2} \mathrm{PPh}_{2} \mathrm{Li} \cdot 2 \mathrm{THF}$. The latter compound was isolated as an unprecedented product from the reaction between the phosphinohydrazine $\left(\mathrm{Ph}_{2} \mathrm{P}\right)_{2} \mathrm{~N}-\mathrm{N}\left(\mathrm{PPh}_{2}\right) \mathrm{H}$ and $\mathrm{LiN}\left(\mathrm{SiMe}_{3}\right)_{2} \cdot{ }^{23}$ Treatment of $\mathrm{Py}_{2} \mathrm{P}\left(\mathrm{NSiMe}_{3}\right)\left(\mathrm{NHSiMe}_{3}\right)$ with lithium reagents $\mathrm{LiN}\left(\mathrm{SiMe}_{2}\right)_{2}$ or $\mathrm{MeLi} / \mathrm{BuLi}$ leads to the cleavage of $\mathrm{P}-$ $\mathrm{N}$ bond to yield $\left[\mathrm{Py}_{2} \mathrm{PNLi}\left(\mathrm{SiMe}_{3}\right)\right]_{2}$ or $\mathrm{Py}_{2} \mathrm{PLi}(\mathrm{THF})_{2}$, respectively. ${ }^{20}$ 
The only structurally characterized iminophosphonamide of $\mathrm{Co}$ (II) reported in the literature is $[\mathrm{Co}\{$ $\left.\left.\mathrm{Ph}_{2} \mathrm{P}\left(\mathrm{N}-p-t \mathrm{BuC}_{6} \mathrm{H}_{4}\right)_{2}\right\}_{2}\right]^{34}$ No complex of $\mathrm{Cu}(\mathrm{II})$ with iminophosphonamides has been reported and only $\mathrm{Cu}(\mathrm{I})$ iminophosphonamide derivatives with a series of olefins as co-ligands have been reported. ${ }^{35,36}$ The discrete ( $\pi$-allyl)nickel(II) iminophosphonamides $\mathrm{Ph}_{2} \mathrm{P}(\mathrm{NR})_{2} \mathrm{Ni}\left(\eta^{3}-\mathrm{C}_{3} \mathrm{H}_{5}\right) \quad\left(\mathrm{R}=\mathrm{SiMe}_{3}\right.$ or $p-\mathrm{Me}-$ $\left.\mathrm{C}_{6} \mathrm{H}_{4}\right),{ }^{37}\left[\mathrm{Ni}\left\{\mathrm{Ph}_{2} \mathrm{P}(\mathrm{NPh})_{2}\right\}_{2}\right]^{38}$ and $\left\{\mathrm{Ph}_{2} \mathrm{P}\left(\mathrm{NSiMe}_{3}\right)_{2}\right\}$ $\mathrm{NiPh}\left(\mathrm{PPh}_{3}\right)^{37}$ are among the structurally characterized heteroleptic iminophosphonamides of $\mathrm{Ni}(\mathrm{II})$. The structurally characterized homoleptic iminophosphonamides of $\mathrm{Ni}(\mathrm{II})$ are $\left.\mathrm{Ni}\left\{i \mathrm{Pr}_{2} \mathrm{P}(\mathrm{NMe})_{2}\right\}_{2}\right]$ and $\left[\mathrm{Ni}\left\{\mathrm{Ph}_{2} \mathrm{P}\left(\mathrm{NSiMe}_{3}\right)_{2}\right\}_{2}\right] .{ }^{39}$

\section{Experimental}

\subsection{General remarks}

All reactions were performed using standard Schlenk and drybox techniques with appropriately dried solvents. All other chemicals used in this study were purchased from commercial sources and used without further purification. The starting arylaminodiphenylphosphine $\mathbf{1 b}^{40,41}$ mesitylazide ${ }^{42,43}$ and the metal silylamides ${ }^{44-46}$ were prepared as previously described. The ${ }^{1} \mathrm{H},{ }^{13} \mathrm{C},{ }^{31} \mathrm{P}$ and ${ }^{7} \mathrm{Li}$ NMR spectra were recorded with Bruker $400 \mathrm{MHz}$ spectrometer, respectively, using TMS, $\mathrm{H}_{3} \mathrm{PO}_{4}(85 \%)$ and $\mathrm{LiCl}$ as the external reference and chemical shift values are reported in ppm.

\section{$2.2 X$-ray crystallography for compounds 1-7}

Single crystal X-ray diffraction data were collected on a Bruker AXS KAPPA APEX-II CCD diffractometer (Monochromatic $\mathrm{MoK} \alpha$ radiation) equipped with Oxford Cryosystem 700 Plus at 100 K. Data collection and unit cell refinement for the data sets were done using the Bruker APPEX-II suite; data reduction and integration were performed by SAINTV 7.685A (Bruker AXS, 2009) and absorption corrections and scaling were done using SADABSV2008/1 (Bruker AXS, 2009). The crystal structures were solved with either OLEX2 or WINGX package using SHELXS-97 and the structure was refined with SHELXL-97 2008. ${ }^{47}$ Single crystal data and refinement results are listed in table 1. All non-hydrogen atoms were refined anisotropically. Hydrogen atoms were fixed at geometrically calculated positions and refined using riding model except the $\mathbf{N H}$ hydrogens of $\mathbf{1}$ and $\mathbf{2}$ that were located from difference Fourier map and refined isotropically. Diamond version $2.1 \mathrm{~d}$ was used to generate graphics for the X-ray structures.

\subsection{General synthetic method for ligands (1 and 2)}

2.3a Synthesis of $\mathrm{P}\left(\mathrm{Ph}_{2}\right) \mathrm{NH}\left(\mathrm{C}_{6} \mathrm{H}_{2}-2,4,6-\mathrm{Me}_{3}\right)$ (1a): A solution of mesitylamine $(6.7 \mathrm{~g}, 50 \mathrm{mmol})$ in $\mathrm{Et}_{2} \mathrm{O}$ $\left(80 \mathrm{~mL}\right.$ ) was cooled to $-78^{\circ} \mathrm{C}$ followed by addition of $n$ BuLi $(32 \mathrm{~mL}, 51 \mathrm{mmol}, 1.6 \mathrm{M})$. The mixture was allowed to cool to room temperature and stirred for $3 \mathrm{~h}$. A solution of $\mathrm{Ph}_{2} \mathrm{PCl}(11.0 \mathrm{~g}, 50 \mathrm{mmol})$ in $\mathrm{Et}_{2} \mathrm{O}$ $(60 \mathrm{~mL})$ was added drop-wise to this solution at $-78^{\circ} \mathrm{C}$. The mixture was stirred at room temperature overnight followed by filtration through Celite and removal of all volatiles under vacuum. The viscous oil thus obtained was washed with pentane to afford a light brown solid that was recrystallized from $\mathrm{Et}_{2} \mathrm{O}$, yield: $13.5 \mathrm{~g} \mathrm{(75 \% )}$; m.p. $112-114^{\circ} \mathrm{C} ;{ }^{1} \mathrm{H}$ NMR $\left(\mathrm{C}_{6} \mathrm{D}_{6}, 400 \mathrm{MHz}\right): \delta=2.10$ (s, $6 \mathrm{H}, o-\mathrm{Me}), 2.15(\mathrm{~s}, 3 \mathrm{H}, p-\mathrm{Me}), 3.55\left(\mathrm{~d}, 1 \mathrm{H}, J_{\mathrm{H}-\mathrm{P}}=\right.$ $8.5 \mathrm{~Hz}, \mathrm{NH}), 6.75$ (s, $2 \mathrm{H}, \mathrm{Ar}-\mathrm{H}), 7.06-7.10$ (m, $6 \mathrm{H}$, Ar-H), 7.47-7.50 (m, $4 \mathrm{H}, \mathrm{Ar}-\mathrm{H}) ;{ }^{13} \mathrm{C}$ NMR $\left(\mathrm{C}_{6} \mathrm{D}_{6}, 100\right.$ $\mathrm{MHz}): \delta=19.32\left(\mathrm{~d}, J_{\mathrm{C}-\mathrm{P}}=6.3 \mathrm{~Hz}\right), 20.77,128.72(\mathrm{~d}$, $\left.J_{\mathrm{C}-\mathrm{P}}=6.4 \mathrm{~Hz}\right), 128.99,129.9\left(\mathrm{~d}, J_{\mathrm{C}-\mathrm{P}}=1.02 \mathrm{~Hz}\right), 130.96$ $\left(\mathrm{d}, J_{\mathrm{C}-\mathrm{P}}=3.6 \mathrm{~Hz}\right), 131.70,131.90,140.83\left(\mathrm{~d}, J_{\mathrm{C}-\mathrm{P}}=14.2\right.$ $\mathrm{Hz}), 143.25\left(\mathrm{~d}, J_{\mathrm{C}-\mathrm{P}}=15.8 \mathrm{~Hz}\right) ;{ }^{31} \mathrm{P}\left\{{ }^{1} \mathrm{H}\right\}$ NMR $\left(\mathrm{C}_{6} \mathrm{D}_{6}\right.$, $162 \mathrm{MHz}): \delta=36$; IR ( $v \mathrm{~cm}^{-1}$, nujol mull): 3362, $3279,1303,1260,1221,1149,1091,1020,851,801$, 738, 722, 696.

2.3b General method for the Synthesis of ligands (1 and 2): A solution of mesitylazide $(40.0 \mathrm{mmol}$, $6.44 \mathrm{~g})$ in THF $(20 \mathrm{~mL})$ was added drop-wise to a THF solution $(40 \mathrm{~mL})$ of the appropriate $(\mathrm{N}-$ diphenylphosphino)-amine (1a or 1b) $(36.0 \mathrm{mmol})$ at $0{ }^{\circ} \mathrm{C}$ under nitrogen atmosphere. The reaction mixture was stirred for $12 \mathrm{~h}$ at room temperature. Removal of all volatiles gave viscous oil which was washed with hexane to give white solid that was recrystallized from THF to afford colourless crystalline solid.

2.3c Synthesis of $\left(2,4,6-\mathrm{Me}_{3} \mathrm{C}_{6} \mathrm{H}_{2} \mathrm{NH}\right) P\left(\mathrm{Ph}_{2}\right)=N\left(\mathrm{C}_{6}\right.$ $\mathrm{H}_{2}-2,4,6-\mathrm{Me}_{3}$ ) (1): Colourless crystalline solid; yield: 13.4 g (74\%); m.p. $166^{\circ} \mathrm{C} ;{ }^{1} \mathrm{H}$ NMR $\left(\mathrm{CDCl}_{3}, 400\right.$ $\mathrm{MHz}): \delta=1.92$ (s, $12 \mathrm{H}, o-\mathrm{Me}), 2.05$ (s, $6 \mathrm{H}, p-\mathrm{Me})$, 4.10 (broad, $1 \mathrm{H}, \mathrm{NH}), 6.59$ (s, $4 \mathrm{H}, m-\mathrm{H}), 7.05-7.10$ (m, $4 \mathrm{H}$, Ar-H), 7.12-7.14 (m, $2 \mathrm{H}, \mathrm{Ar}-\mathrm{H}), 7.62-7.67$ $(\mathrm{m}, 4 \mathrm{H}, \mathrm{Ar}-\mathrm{H}) ;{ }^{13} \mathrm{C} \mathrm{NMR}\left(\mathrm{CDCl}_{3}, 100 \mathrm{MHz}\right): \delta=$ 20.44 (br), 20.54, 127.62 (d, $\left.J_{\mathrm{C}-\mathrm{P}}=24.3 \mathrm{~Hz}\right), 127.85$, $128,128.85$ (br), 130.80 (d, $\left.J_{\mathrm{C}-\mathrm{P}}=2.8 \mathrm{~Hz}\right), 131.76(\mathrm{~d}$, $\left.J_{\mathrm{C}-\mathrm{P}}=9.8 \mathrm{~Hz}\right), 134.15,135.41 ;{ }^{31} \mathrm{P}\left\{{ }^{1} \mathrm{H}\right\} \mathrm{NMR}\left(\mathrm{CDCl}_{3}\right.$, $162 \mathrm{MHz}): \delta=-13.04$; EI-MS: $m / z: 453\left[\mathrm{M}^{+}\right]$; IR $(v$ $\mathrm{cm}^{-1}$, nujol mull): $3340,1435,1306,1279,1245,1214$, 1182, 1161, 1118, 1058, 1024, 997, 946, 883, 853, 769, 750, 711, 697, 600. Anal. Calcd. in (\%) for $\mathrm{C}_{30} \mathrm{H}_{33} \mathrm{~N}_{2} \mathrm{P}$; 
Table 1. Crystallographic data and refinement parameters for compounds 1-7.

\begin{tabular}{|c|c|c|c|c|c|c|c|}
\hline Compound $^{[\mathrm{a}]}$ & 1 & $2 \cdot 0.5\left(\mathrm{C}_{4} \mathrm{H}_{8} \mathrm{O}\right)$ & 3 & 4 & 5 & $6 \cdot 0.5 \mathrm{C}_{7} \mathrm{H}_{8}$ & $7 \cdot 0.5 \mathrm{C}_{7} \mathrm{H}_{8}$ \\
\hline Formula & $\mathrm{C}_{30} \mathrm{H}_{33} \mathrm{~N}_{2} \mathrm{P}$ & $\begin{array}{c}\mathrm{C}_{33} \mathrm{H}_{39} \mathrm{~N}_{2} \mathrm{P} \cdot 0.5 \\
\left(\mathrm{C}_{4} \mathrm{H}_{8} \mathrm{O}\right)\end{array}$ & $\begin{array}{c}\mathrm{C}_{30} \mathrm{H}_{32} \mathrm{~N}_{2} \mathrm{PLi} \\
\text { tmeda }\end{array}$ & $\begin{array}{c}\mathrm{C}_{33} \mathrm{H}_{38} \mathrm{~N}_{2} \mathrm{PLi} \cdot \\
\text { tmeda }\end{array}$ & $\mathrm{C}_{60} \mathrm{H}_{64} \mathrm{~N}_{4} \mathrm{P}_{2} \mathrm{Co}$ & $\begin{array}{c}\mathrm{C}_{60} \mathrm{H}_{64} \mathrm{~N}_{4} \mathrm{P}_{2} \mathrm{Cu} \cdot \\
0.5 \mathrm{C}_{7} \mathrm{H}_{8}\end{array}$ & $\begin{array}{c}\mathrm{C}_{60} \mathrm{H}_{64} \mathrm{~N}_{4} \mathrm{P}_{2} \mathrm{Ni} \\
0.5 \mathrm{C}_{7} \mathrm{H}_{8}\end{array}$ \\
\hline fw, $\mathrm{gmol}^{-1}$ & 452.6 & 530.7 & 574.7 & 616.8 & 962.1 & 1050.8 & 1045.9 \\
\hline Crystal system & Monoclinic & Monoclinic & Monoclinic & Monoclinic & Triclinic & Triclinic & Triclinic \\
\hline Space group & $P 2_{1} / c$ & $P 2{ }_{1} / c$ & $P 2_{1} / n$ & $P 2{ }_{1} / c$ & $P-1$ & $P-1$ & $P-1$ \\
\hline$T / \mathrm{K}$ & 100 & 100 & 100 & 100 & 100 & 100 & 100 \\
\hline$a / \AA$ & $12.014(2)$ & $12.818(1)$ & $9.575(1)$ & $10.060(1)$ & $12.088(1)$ & $9.906(1)$ & $11.182(1)$ \\
\hline$b / \AA$ & $15.537(2)$ & $18.166(2)$ & $18.389(1)$ & $14.962(1)$ & $13.185(2)$ & $12.054(2)$ & $11.225(2)$ \\
\hline$c / \AA$ & $18.174(2)$ & $25.979(2)$ & $18.839(1)$ & $23.912(2)$ & $17.062(2)$ & $12.615(2)$ & $11.573(2)$ \\
\hline$\alpha /^{\circ}$ & 90 & 90 & 90 & 90 & $87.50(1)$ & $82.02(1)$ & $76.26(1)$ \\
\hline$\beta /^{\circ}$ & $132.97(1)$ & $90.26(1)$ & $90.43(1)$ & $92.57(1)$ & $69.48(2)$ & $80.76(2)$ & $86.14(2)$ \\
\hline$\gamma /{ }^{\circ}$ & 90 & 90 & 90 & 90 & $88.15(2)$ & $66.87(2)$ & $89.85(2)$ \\
\hline$V / \AA^{3}$ & $2482.27(2)$ & $6049.14(6)$ & $3317.01(1)$ & $3595.68(2)$ & $2544.03(1)$ & $1362.60(2)$ & $1407.75(4)$ \\
\hline$Z$ & 4 & 8 & 4 & 4 & 2 & 1 & 1 \\
\hline$\rho_{c} / \mathrm{g} \cdot \mathrm{cm}^{-3}$ & 1.21 & 1.17 & 1.15 & 1.14 & 1.26 & 1.28 & 1.23 \\
\hline$\mu\left(\mathrm{Mo}-K_{\alpha}\right) / \mathrm{mm}^{-1}$ & 0.131 & 0.118 & 0.113 & 0.108 & 0.443 & 0.506 & 0.446 \\
\hline reflections collected & 15384 & 32165 & 19787 & 25973 & 20123 & 9308 & 11480 \\
\hline independent reflections & 4373 & 10633 & 5867 & 6353 & 8959 & 4768 & 4881 \\
\hline data/restraints/parameters & $4373 / 0 / 308$ & $10633 / 0 / 714$ & $5867 / 0 / 389$ & $6353 / 0 / 417$ & $8959 / 0 / 616$ & $4768 / 0 / 343$ & $4881 / 0 / 355$ \\
\hline$R 1, w R 2[I>2 \sigma(I)]^{[\mathrm{a}]}$ & $0.036,0.084$ & $0.061,0.156$ & $0.035,0.082$ & $0.036,0.084$ & $0.066,0.101$ & $0.066,0.137$ & $0.039,0.088$ \\
\hline R1, wR2 (all data)[a] & $0.049,0.089$ & $0.076,0.169$ & $0.046,0.087$ & $0.054,0.090$ & $0.162,0.127$ & $0.123,0.162$ & $0.062,0.096$ \\
\hline $\mathrm{GOF}$ & 1.031 & 1.055 & 1.026 & 0.968 & 0.952 & 1.022 & 1.01 \\
\hline
\end{tabular}

[a] $R 1=\Sigma\|F \mathrm{O}|--| F \mathrm{c}\| / \Sigma|F \mathrm{O}| . w R 2=\left[\Sigma w\left(\left|F_{\mathrm{O}}{ }^{2}\right|--\left|F \mathrm{c}^{2}\right|\right)^{2} / \Sigma w\left|F \mathrm{O}^{2}\right|^{2}\right]^{1 / 2}$ 
C, 79.62; H, 7.35; N, 6.19. Found: C, 79.67; H, 7.32; N, 6.25 .

2.3d Synthesis of (2,6-iPr $\left.\mathrm{C}_{6} \mathrm{H}_{3} \mathrm{NH}\right) \mathrm{P}\left(\mathrm{Ph}_{2}\right)=\mathrm{N}\left(\mathrm{C}_{6}\right.$ $\mathrm{H}_{2}-2,4,6-\mathrm{Me}_{3}$ ) (2): Colourless crystalline solid; yield: $14.24 \mathrm{~g}(72 \%)$; m.p. $135^{\circ} \mathrm{C} .{ }^{1} \mathrm{H}$ NMR $\left(\mathrm{C}_{6} \mathrm{D}_{6}, 400 \mathrm{MHz}\right)$ : $\delta=1.14\left(\mathrm{~d}, 12 \mathrm{H}, J_{\mathrm{H}-\mathrm{H}}=6.7 \mathrm{~Hz}, \mathrm{CH} M e_{2}\right), 2.21(\mathrm{~s}$, $3 \mathrm{H}, p-M e$ ), 2.26 (s, $6 \mathrm{H}, o-M e), 3.43$ (broad, $2 \mathrm{H}$, $\mathrm{CH} \mathrm{Me}_{2}$ ), 4.13 (broad, $\left.1 \mathrm{H}, \mathrm{NH}\right), 6.80$ (s, $2 \mathrm{H}, \mathrm{Ar}-$ H), 7.07-7.26 (m, 9 H, Ar-H), 7.80-7.86 (m, 4 H, Ar$\mathrm{H}) ;{ }^{13} \mathrm{C}$ NMR $\left(\mathrm{C}_{6} \mathrm{D}_{6}, 100 \mathrm{MHz}\right): \delta=20.60,20.72$, 23.94, (d, $\left.J_{\mathrm{C}-\mathrm{P}}=13.7 \mathrm{~Hz}\right), 28.94\left(\mathrm{~d} J_{\mathrm{C}-\mathrm{P}}=15 \mathrm{~Hz}\right)$, $119.79\left(\right.$ br d $\left.J_{\mathrm{C}-\mathrm{P}}=13 \mathrm{~Hz}\right), 123.13\left(\mathrm{~d} J_{\mathrm{C}-\mathrm{P}}=24 \mathrm{~Hz}\right)$, 127.7-128.2 (complex multiplets partially hidden by the solvent resonance), $129.68\left(\mathrm{~d} J_{\mathrm{C}-\mathrm{P}}=14 \mathrm{~Hz}\right), 131$ (br), 132.18 (vtr, $J_{\mathrm{C}-\mathrm{P}}=23 \mathrm{~Hz}$ ), 134.5 (br), 135.29 (br), 141.55 (br), 143.84 (br); ${ }^{31} \mathrm{P}\left\{{ }^{1} \mathrm{H}\right\}$ NMR $\left(\mathrm{C}_{6} \mathrm{D}_{6}, 162\right.$ MHz): $\delta=-15.34$; EI-MS: $m / z: 495\left[M^{+}\right], 378\left[\mathrm{M}^{+}-\right.$ $\left.\mathrm{Me}_{3} \mathrm{C}_{6} \mathrm{H}_{2}\right], 336\left[\mathrm{M}^{+}-i \mathrm{Pr}_{2} \mathrm{C}_{6} \mathrm{H}_{3}\right]$; IR ( $v \mathrm{~cm}^{-1}$, nujol mull): 3362, 1303, 1254, 1217, 1147, 1105, 1068, 1026, 956, 936, 904, 849, 792, 744, 722, 695. Anal. Calcd. in (\%) for $\mathrm{C}_{33} \mathrm{H}_{39} \mathrm{~N}_{2} \mathrm{P} ; \mathrm{C}, 80.13 ; \mathrm{H}, 7.95 ; \mathrm{N}, 5.66$. Found: C, 79.15; H, 7.90; N, 5.51.

\subsection{General synthetic method for lithium derivatives (3 and 4)}

A solution of ligand ( $\mathbf{1}$ or $\mathbf{2})(1.0 \mathrm{mmol})$ and tmeda $(0.5$ $\mathrm{mL})$ in toluene $(20 \mathrm{~mL})$ was cooled to $-78^{\circ} \mathrm{C}$. To it was added $n \operatorname{BuLi}(0.7 \mathrm{~mL}, 1.1 \mathrm{mmol}, 1.6 \mathrm{M})$ and allowed to cool to room temperature and stirring was continued for $6 \mathrm{~h}$. Concentration of the mother liquor to $5 \mathrm{~mL}$ gave the lithium derivative as colourless crystals at $-30^{\circ} \mathrm{C}$ in 2 days.

2.4a Synthesis of (Li.tmeda)[(2,4,6- $\left.\mathrm{Me}_{3} \mathrm{C}_{6} \mathrm{H}_{2}\right) \mathrm{NP}$ $\left.\left(\mathrm{Ph}_{2}\right)=\mathrm{N}\left(\mathrm{C}_{6} \mathrm{H}_{2}-2,4,6-\mathrm{Me}_{3}\right)\right]$ (3): Colourless crystalline solid; yield $0.38 \mathrm{~g}(66 \%)$; m.p. $145^{\circ} \mathrm{C}$ (decomposition); ${ }^{1} \mathrm{H}$ NMR (400 MHz, $\left.\mathrm{C}_{6} \mathrm{D}_{6}\right): \delta=1.81(\mathrm{~s}, 4 \mathrm{H}$, $\mathrm{N}-\mathrm{CH}_{2}$ ), 1.92 (s, $12 \mathrm{H}, \mathrm{N}-\mathrm{Me}$ ), 2.24-2.26 (overlapped singlet, $18 \mathrm{H}, o-\& p-M e), 6.91(\mathrm{~s}, 4 \mathrm{H}, \mathrm{Ar}-\mathrm{H}), 6.99-$ $7.04(\mathrm{~m}, 6 \mathrm{H}, \mathrm{Ar}-\mathrm{H}), 7.67-7.72(\mathrm{~m}, 4 \mathrm{H}, \mathrm{Ar}-\mathrm{H}) ;{ }^{13} \mathrm{C}$ $\operatorname{NMR}\left(100 \mathrm{MHz}, \mathrm{C}_{6} \mathrm{D}_{6}\right): \delta=20.99\left(\mathrm{~d}, J_{\mathrm{C}-\mathrm{P}}=4.3 \mathrm{~Hz}\right)$, $22.06\left(\mathrm{~d}, J_{\mathrm{C}-\mathrm{P}}=4.1 \mathrm{~Hz}\right), 45.39,56.51,126.93(\mathrm{br})$, 127.32 (overlapped doublets), 128.96 (br), 129.32 (br), $131.66\left(\right.$ vtr, $\left.J_{\mathrm{C}-\mathrm{P}}=12.7 \mathrm{~Hz}\right), 133.37\left(\mathrm{vtr}, J_{\mathrm{C}-\mathrm{P}}=11.6 \mathrm{~Hz}\right)$, $142.15\left(\mathrm{dd}, J_{\mathrm{C}-\mathrm{P}}=92 \& 4.9 \mathrm{~Hz}\right), 147.73\left(\mathrm{~d}, J_{\mathrm{C}-\mathrm{P}}=3.6 \mathrm{~Hz}\right)$; ${ }^{31} \mathrm{P}\left\{{ }^{1} \mathrm{H}\right\}$ NMR $\left(162 \mathrm{MHz}, \mathrm{C}_{6} \mathrm{D}_{6}\right): \delta=-8.34 ;{ }^{7} \mathrm{Li} \mathrm{NMR}$ (155 MHz, $\left.\mathrm{C}_{6} \mathrm{D}_{6}\right): \delta=1.18$; IR $\left(v \mathrm{~cm}^{-1}\right.$, nujol mull): 1434, 1375, 1309, 1267, 1158, 1111, 1099, 1032, 943, 852, 794, 760, 726, 704, 656, 584, 535, 525, 497, 455.

2.4b Synthesis of (Li.tmeda)[(2,6-i $\left.\mathrm{Pr}_{2} \mathrm{C}_{6} \mathrm{H}_{3}\right) \mathrm{NP}$ $\left.\left(P h_{2}\right)=N\left(C_{6} H_{2}-2,4,6-M e_{3}\right)\right](4)$ : Colourless crystal- line solid; yield: $0.38 \mathrm{~g}$ (62\%); m.p. $182{ }^{\circ} \mathrm{C} ;{ }^{1} \mathrm{H}$ NMR $\left(400 \mathrm{MHz}, \mathrm{C}_{6} \mathrm{D}_{6}\right): \delta=1.07\left(\mathrm{~d}, J_{\mathrm{H}-\mathrm{H}}=6.8 \mathrm{~Hz}, 12\right.$ $\mathrm{H}, \mathrm{CHMe}$ ), 1.70 (broad, $4 \mathrm{H}, \mathrm{N}-\mathrm{CH}_{2}$ ), 1.92 (s, 12 $\mathrm{H}, \mathrm{N}-M e), 2.16$ (s, $6 \mathrm{H}, o-M e), 2.20$ (s, $3 \mathrm{H}, p-M e)$, 4.10 (sept, $6.8 \mathrm{~Hz}, 2 \mathrm{H}, \mathrm{CHMe}$ ), 6.94 (s, $2 \mathrm{H}, \mathrm{Ar}-\mathrm{H}$ ), 6.95-7.04 (m, 7 H, Ar-H), 7.20-7.22 (m, 2H, Ar-H), 7.53-7.57 (m, $4 \mathrm{H}, \mathrm{Ar}-\mathrm{H}) ;{ }^{13} \mathrm{C}$ NMR (100 MHz, $\left.\mathrm{C}_{6} \mathrm{D}_{6}\right)$ : $\delta=21.04\left(\mathrm{~d}, J_{\mathrm{C}-\mathrm{P}}=3 \mathrm{~Hz}\right), 21.70\left(\mathrm{~d}, J_{\mathrm{C}-\mathrm{P}}=3 \mathrm{~Hz}\right), 24.76$ (d, $\left.J_{\mathrm{C}-\mathrm{P}}=3.5 \mathrm{~Hz}\right), 28.40(\mathrm{br}), 45.50(\mathrm{br}), 56.72(\mathrm{br})$, 120.04, 123.53, 127.19-127.34 (overlapped doublets), $128.95,129.40,131.57$ (br), 133.95 (vtr, $J_{\mathrm{C}-\mathrm{P}}=10.3$ $\mathrm{Hz}), 142.30\left(\mathrm{dd}, J_{\mathrm{C}-\mathrm{P}}=92.2 \& 4.6 \mathrm{~Hz}\right), 144.06$ (vtr, $\left.J_{\mathrm{C}-\mathrm{P}}=9.2 \mathrm{~Hz}\right), 147.09\left(\mathrm{~d}, J_{\mathrm{C}-\mathrm{P}}=2.2 \mathrm{~Hz}\right), 147.82$ (br); ${ }^{31} \mathrm{P}\left\{{ }^{1} \mathrm{H}\right\}$ NMR $\left(162 \mathrm{MHz}, \mathrm{C}_{6} \mathrm{D}_{6}\right): \delta=-11.01 ;{ }^{7} \mathrm{Li}$ NMR $\left(155 \mathrm{MHz}, \mathrm{C}_{6} \mathrm{D}_{6}\right): \delta=0.92$; IR $\left(v, \mathrm{~cm}^{-1}\right.$, nujol mull): = 1376, 1307, 1261, 1219, 1151, 1097, 1025, 945, 851, 796, 747, 697, 584, 520, 454.

\subsection{General synthetic method for metal complexes 5 and 6}

$\mathrm{M}\left[\mathrm{N}\left(\mathrm{SiMe}_{3}\right)_{2}\right]_{2}$ was generated in situ by $\mathrm{MCl}_{2}(0.4$ $\mathrm{mmol})$ and $\mathrm{LiN}\left(\mathrm{SiMe}_{3}\right)_{2}(0.8 \mathrm{mmol}) 10 \mathrm{~mL}$ dry THF in a $50 \mathrm{~mL}$ Schlenk flask. To it was added a solution of $\mathbf{1}$ $(0.34 \mathrm{~g}, 0.75 \mathrm{mmol})$ in $10 \mathrm{~mL} \mathrm{THF}$ at $-78^{\circ} \mathrm{C}$. The mixture was stirred overnight at ambient temperature. All volatiles were removed under vacuum and the product was extracted in dry toluene $(20 \mathrm{~mL})$. The filtrate was concentrated to about $8 \mathrm{~mL}$ and stored at $-30^{\circ} \mathrm{C}$ to give crystals of the metal complexes.

2.5a $\mathrm{Co}\left[\mathrm{Ph}_{2} \mathrm{P}\left(\mathrm{NC}_{6} \mathrm{H}_{2}-2,4,6-\mathrm{Me}_{3}\right)_{2}\right]_{2}$ (5): Blue crystalline solid; yield: $0.54 \mathrm{~g}(74 \%)$; m.p. $186^{\circ} \mathrm{C}$. EI$\operatorname{MS}(m / z): 961\left[M^{+}\right], 843\left[\mathrm{M}^{+}-\mathrm{Me}_{3} \mathrm{C}_{6} \mathrm{H}_{2}\right], 776\left[\mathrm{M}^{+}-\right.$ $\left.\mathrm{C}_{6} \mathrm{H}_{5}\right], 591\left[\mathrm{M}^{+}-2 \mathrm{P}\left(\mathrm{C}_{6} \mathrm{H}_{5}\right)_{2}\right], 511\left[\mathrm{M}^{+}-\mathrm{C}_{30} \mathrm{H}_{31} \mathrm{~N}_{2} \mathrm{P}\right]$; IR ( $v \mathrm{~cm}^{-1}$, nujol mull): 1307, 1256, 1215, 1162, 1075, $853,802,751,722,697,659,578,539$.

2.5b Cu $\left.\mathrm{Ph} \mathrm{h}_{2} \mathrm{P}\left(\mathrm{NC}_{6} \mathrm{H}_{2}-2,4,6-\mathrm{Me}_{3}\right)_{2}\right]_{2} \quad$ (6): Reddish brown crystalline solid; yield: $0.58 \mathrm{~g}$ (83 \%); m.p. $157^{\circ} \mathrm{C}$; EI-MS $(m / z): 967\left[M^{+}\right], 951\left[M^{+}-\mathrm{Me}\right], 727$ $\left[\mathrm{M}^{+}-2 \mathrm{Me}_{3} \mathrm{C}_{6} \mathrm{H}_{2}\right], 693\left[\mathrm{M}^{+}-\mathrm{Me}_{3} \mathrm{C}_{6} \mathrm{H}_{2} \& 2 \mathrm{C}_{6} \mathrm{H}_{5}\right]$, $514\left[\mathrm{M}^{+}-\mathrm{C}_{30} \mathrm{H}_{31} \mathrm{~N}_{2} \mathrm{P}\right]$; IR ( $v \mathrm{~cm}^{-1}$, nujol mull): 1307, 1259, 1159,1102, 1019, 848, 799, 746, 720, 710, 696, $660,582,525$.

2.5c $\mathrm{Ni}\left[\mathrm{Ph}_{2} \mathrm{P}\left(\mathrm{NC}_{6} \mathrm{H}_{2}-2,4,6-\mathrm{Me}_{3}\right)_{2}\right]_{2}$ (7): A solution of $1(0.45 \mathrm{~g}, 1.0 \mathrm{mmol})$ in dry toluene $(10 \mathrm{~mL})$ was cooled to $-78^{\circ} \mathrm{C}$; to this solution $n \operatorname{BuLi}(0.75 \mathrm{~mL}, 1.2$ mmol, $1.6 \mathrm{M}$ ) was added and the solution was allowed to cool to room temperature with further stirring for $2 \mathrm{~h}$. This solution was added via a cannula to a suspension of $\left(\mathrm{PPh}_{3}\right)_{2} \mathrm{NiCl}_{2}(0.33 \mathrm{~g}, 0.5 \mathrm{mmol})$ in $15 \mathrm{~mL}$ toluene. The 
colour slowly changed to greenish yellow. It was stirred overnight at ambient temperature followed by filtration through Celite and concentration to $10 \mathrm{~mL}$. Storage of this solution at $-30^{\circ} \mathrm{C}$ gave reddish brown crystals after one week, yield: $0.73 \mathrm{~g}(76 \%)$; m.p. $240{ }^{\circ} \mathrm{C} ;{ }^{1} \mathrm{H}$ NMR (400 MHz, $\left\{d_{8}\right\}$ THF): $\delta=1.67$ (s, $12 \mathrm{H}, p$-Me), 2.032.09 (merged singlets, $24 \mathrm{H}, o-M e), 6.48(\mathrm{~s}, 4 \mathrm{H}, \mathrm{Ar}-\mathrm{H})$, 6.61 (s, 4 H, Ar-H), 7.28-7.32 (m, 8 H, Ar-H), 7.357.39 (m, $4 \mathrm{H}, \mathrm{Ar}-\mathrm{H}), 7.76-7.81$ (m, $8 \mathrm{H}, \mathrm{Ar}-\mathrm{H}) ;{ }^{31} \mathrm{P}\left\{{ }^{1} \mathrm{H}\right\}$ NMR (162 MHz, $\left.\mathrm{C}_{6} \mathrm{D}_{6}\right): \delta=-16.83$; EI-MS $(m / z)$ : $962\left[M^{+}\right], 948\left[M^{+}-\mathrm{Me}\right], 723\left[\mathrm{M}^{+}-2 \mathrm{Me}_{3} \mathrm{C}_{6} \mathrm{H}_{2}\right], 652$ $\left[\mathrm{M}^{+}-4\left(\mathrm{C}_{6} \mathrm{H}_{5}\right)_{2}\right], 590\left[\mathrm{M}^{+}-2 \mathrm{P}\left(\mathrm{C}_{6} \mathrm{H}_{5}\right)_{2}\right], 509\left[\mathrm{M}^{+}-\right.$ $\left.\mathrm{C}_{30} \mathrm{H}_{31} \mathrm{~N}_{2} \mathrm{P}\right]$; IR $\left(v \mathrm{~cm}^{-1}\right.$, nujol mull): 1307, 1258, 1214, 1150, 996, 887, 852, 782, 747, 722, 696, 537.

\section{Results and Discussion}

Arylaminodiphenylphosphines $\mathrm{Ph}_{2}$ PNHAr $(\mathrm{Ar}=2,4,6$ $\mathrm{Me}_{3} \mathrm{C}_{6} \mathrm{H}_{2}$ 1a; $\mathrm{Ar}=2,6-i \mathrm{Pr}_{2} \mathrm{C}_{6} \mathrm{H}_{3}$ 1b) were synthesized from ArNHLi and $\mathrm{ClPPh}_{2}$ in $\mathrm{Et}_{2} \mathrm{O}$ and purified by recrystallization from $\mathrm{Et}_{2} \mathrm{O} .{ }^{40,41}$ The ${ }^{31} \mathrm{P}\left\{{ }^{1} \mathrm{H}\right\} \mathrm{NMR}$ spectrum of 1a shows a single resonance at $36 \mathrm{ppm}$ and the corresponding signal for $\mathbf{1 b}$ appears at 45.5 ppm. Ligands $\mathbf{1}$ and $\mathbf{2}$ have been prepared by Staudinger type reaction using mesitylazide following the synthetic route outlined in scheme 1 . These iminophosphonamine ligands have been characterized by ${ }^{1} \mathrm{H},{ }^{13} \mathrm{C}$ and ${ }^{31} \mathrm{P}\left\{{ }^{1} \mathrm{H}\right\}$ NMR spectroscopy, EI mass spectrometry, and single crystal $\mathrm{X}$-ray structural analysis. The ${ }^{1} \mathrm{H}$ NMR spectrum of 1 reveals $\mathrm{NH}$ at $4.10 \mathrm{ppm}$ and two singlets that appear at 1.92 and $2.05 \mathrm{ppm}$ correspond to $o-$ and $p-\mathrm{Me}$ of the mesityl group. The aromatic $m-\mathrm{H}$ appears as a singlet at $6.59 \mathrm{ppm}$. Other aromatic hydrogen atoms of $\mathbf{1}$ appear as multiplets between 7.05-7.10 and 7.12-7.14 ppm. The ${ }^{31} \mathrm{P}\left\{{ }^{1} \mathrm{H}\right\}$ NMR spectrum for this compound shows a single resonance at $-13.04 \mathrm{ppm}$, an up-field shift compared to the precursor 1a. The base peak in the EI mass spectrum of 1 appeared at $m / z 453$ that also corresponds to the molecular ion. The NH stretch in $\mathbf{1}$ was observed at $3340 \mathrm{~cm}^{-1}$. Single crystals of 1 suitable for $\mathrm{X}$-ray structure analysis were grown from toluene at $-30^{\circ} \mathrm{C}$ after several days. Compound $\mathbf{1}$ crystallizes in monoclinic system with $P 2{ }_{1} / c$ space group (table 1 ). In solid state structure of $\mathbf{1}$, the central $\mathrm{P}$ atom shows distorted tetrahedron geometry (figure 1). The NPN backbone of $\mathbf{1}$ is arranged in a plane and the phenyl rings on central $\mathrm{P}$ atom are arranged above and below this plane. The two aryl substituents on the $\mathrm{N}$ atoms are mutually transoid. The amine N(1)-P(1) bond length is 1.675(1) $\AA$, whereas the imine bond $\mathrm{N}(2)-\mathrm{P}(1)(1.538(1) \AA)$ is shorter than $\mathrm{N}(1)-\mathrm{P}(1)$ and these bond lengths are similar to that reported for $\left[\mathrm{Py}_{2} \mathrm{P}\left(\mathrm{NSiMe}_{3}\right)\left(\mathrm{NHSiMe}_{3}\right)\right] .{ }^{20}$

The ${ }^{1} \mathrm{H}$ NMR features of compound $\mathbf{2}$ are as expected, showing the $\mathrm{NH}$ at $4.13 \mathrm{ppm}$ and a broad septet at $3.43 \mathrm{ppm}$ for $\mathrm{CH} \mathrm{Me}_{2}$. The $\mathrm{CHMe}$ appear as a doublet at $1.14 \mathrm{ppm}$ and the respective $o-$ and $p-$ Me of mesityl groups are seen at 2.26 and $2.21 \mathrm{ppm}$. The aromatic $m-\mathrm{H}$ appears as a singlet at $6.80 \mathrm{ppm}$. Other aromatic hydrogen atoms of $\mathbf{2}$ appear as complex multiplets between 7.07-7.26 and 7.80-7.86 ppm. The ${ }^{31} \mathrm{P}\left\{{ }^{1} \mathrm{H}\right\}$ NMR spectrum of 2 shows a single resonance at $-15.34 \mathrm{ppm}$, which is shifted up-field compared to its precursor $\mathbf{1 b}(45.5 \mathrm{ppm})$. The most abundant ion as well as the molecular ion in the EI mass spectrum of 2 appeared at $m / z$ 495. The FT-IR spectrum of $\mathbf{2}$ showed the NH stretch at $3362 \mathrm{~cm}^{-1}$.

Compound 2 crystallizes in monoclinic system with $P 2_{1} / c$ space group (table 1 ). The central $\mathrm{P}$ atom in compound 2 resides in tetrahedral environment similar to that of 1 (figure 2). The $\mathrm{N}(1)-\mathrm{P}(1)$ and $\mathrm{N}(2)-\mathrm{P}(1)$ bond lengths are $1.657(1)$ and $1.536(1) \AA$, respectively, similar to that of $\mathbf{1}$. The $\mathrm{N}(1)-\mathrm{P}(1)-\mathrm{N}(2), \mathrm{N}(1)-\mathrm{P}(1)-$ $\mathrm{C}(28), \mathrm{C}(28)-\mathrm{P}(1)-\mathrm{C}(22)$ and $\mathrm{C}(28)-\mathrm{P}(1)-\mathrm{N}(2)$ bond

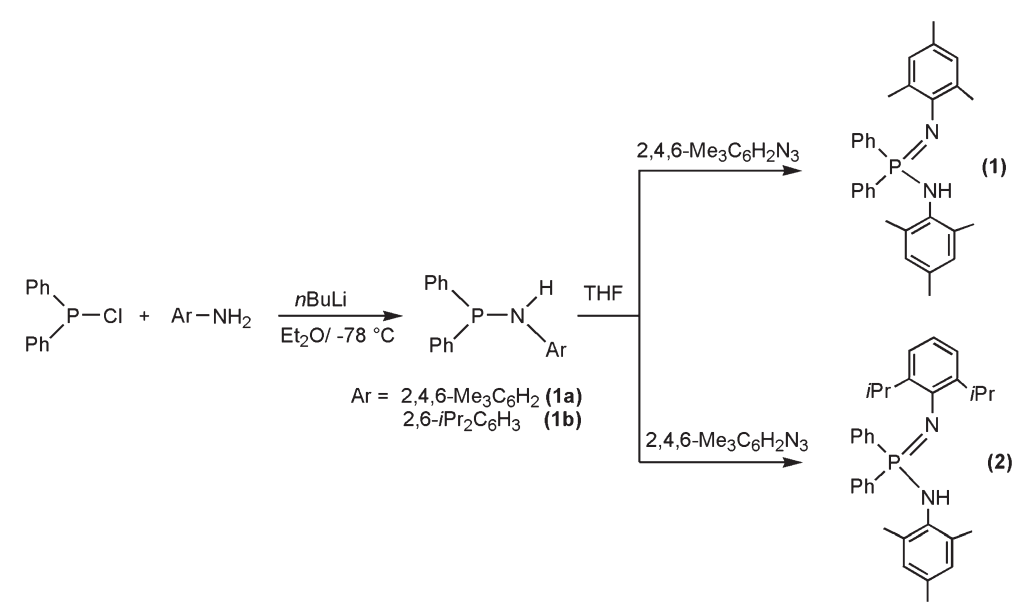

Scheme 1. Synthesis of sterically congested iminophosphonamine ligands $\mathbf{1}$ and $\mathbf{2}$. 


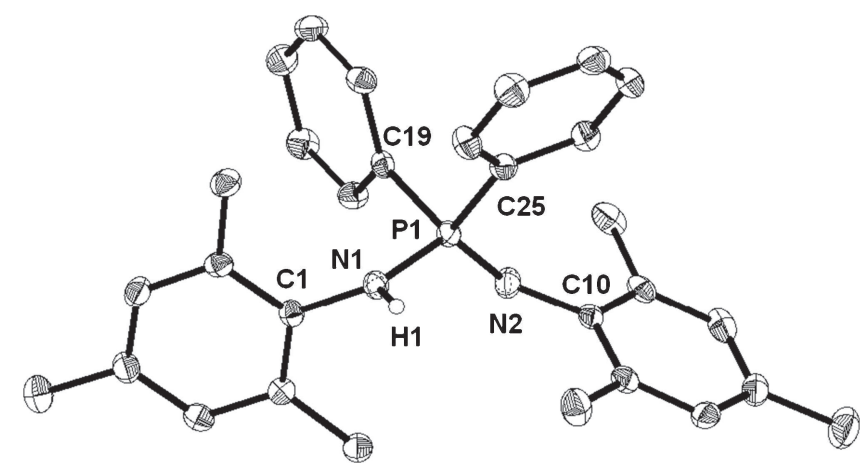

Figure 1. Single crystal X-ray structure of 1. All hydrogen atoms, except that on $\mathrm{N}(1)$ are omitted for clarity. Thermal ellipsoids are drawn at $50 \%$ probability. Selected bond lengths $(\AA)$ and bond angles $\left({ }^{\circ}\right)$ : $\mathrm{N}(1)-\mathrm{P}(1)$ $1.675(1), \mathrm{N}(2)-\mathrm{P}(1) \quad 1.538(1), \mathrm{N}(1)-\mathrm{C}(1)$ 1.444(1), N(2)C(10) 1.393(1); N(1)-P(1)-N(2) 120.90(1), C(1)-N(1)-P(1) 126.31(2), C(10)-N(2)-P(1) 142.51(1).

angle, respectively, 109.60(1), 110.13(2), 104.93(2), $114.20(1)^{\circ}$ are close to tetrahedral bond angles; whereas the other angles $\left\{\mathrm{C}(22)-\mathrm{P}(1)-\mathrm{N}(2) 118.10(1)^{\circ}\right.$ and $\left.\mathrm{N}(1)-\mathrm{P}(1)-\mathrm{C}(22) 98.69(1)^{\circ}\right\}$ deviate slightly from the regular tetrahedral angle.

Treatment of $\mathbf{1}$ and $\mathbf{2}$ with $n \mathrm{BuLi}$ in toluene in the presence of tmeda (tetramethylethylenediamine)

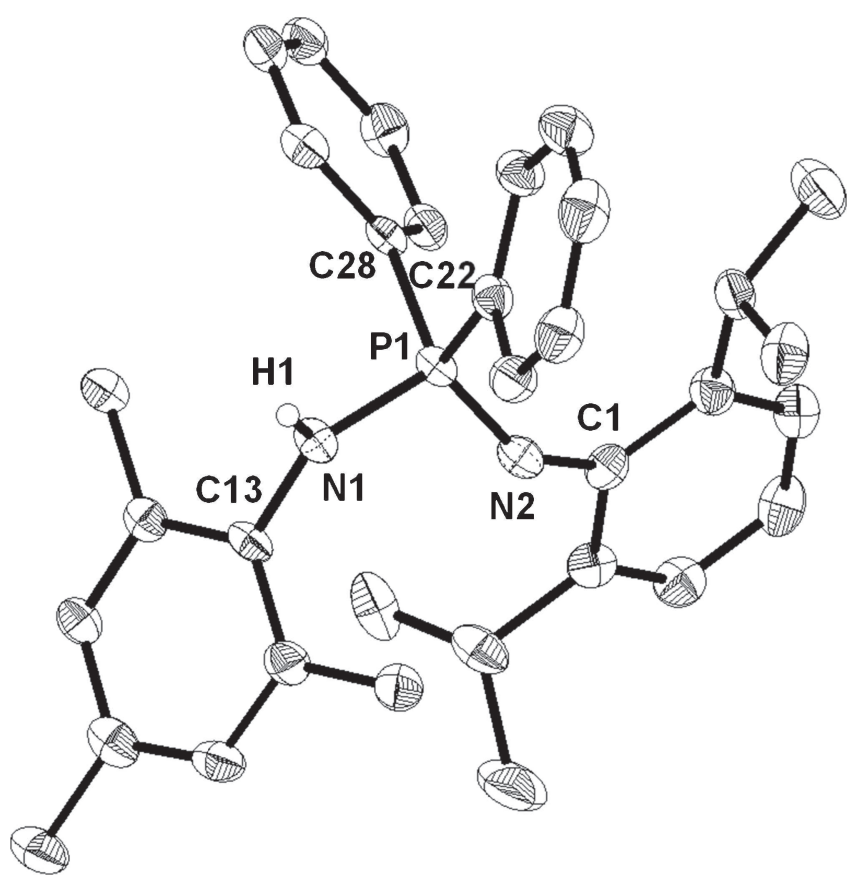

Figure 2. Molecular structure of 2. All hydrogen atoms, except that on N(1), and THF molecule in the lattice are omitted for clarity. Thermal ellipsoids are drawn at $50 \%$ probability. Selected bond lengths $(\AA)$ and bond angles $\left({ }^{\circ}\right)$ : N (1)-P(1) $1.657(1), \mathrm{N}(2)-\mathrm{P}(1) 1.536(1), \mathrm{N}(1)-\mathrm{C}(13)$ 1.441(1), N(2)C(1) 1.396(1); N(1)-P(1)-N(2) 109.60(1), N(1)-P(1)-C(28) 110.13(2), C(22)-P(1)-N(2) 118.10(1), C(13)-N(1)-P(1) 127.64(2), C(1)-N(2)-P(1) 143.85(1), C(28)-P(1)-C(22) 104.93(2), C(28)-P(1)-N(2) 114.20(1). afforded the corresponding tmeda adduct of highly moisture sensitive lithium derivatives $\mathbf{3}$ and $\mathbf{4}$ (scheme 2); and as observed in ${ }^{7} \mathrm{Li} \mathrm{NMR}$, the chelates are highly stable and no loss of tmeda was observed even after drying under vacuum for several hours. Complexes $\mathbf{3}$ and $\mathbf{4}$ are the first examples of NPNLi moiety with tmeda chelate, the evidence for tmeda chelate was observed in the ${ }^{1} \mathrm{H}$ and ${ }^{13} \mathrm{C}$ NMR and was confirmed by single crystal X-ray structure of $\mathbf{3}$ and $\mathbf{4}$.

The ${ }^{1} \mathrm{H}$ NMR spectra of $\mathbf{3}$ and $\mathbf{4}$ show the absence of $\mathrm{NH}$ signals, tmeda $\mathrm{NCH}_{2}$ appears as a singlet at 1.81 and $\mathrm{NCH}_{3}$ of the same is observed as a singlet at 1.92 for $\mathbf{3}$ (the corresponding signals of tmeda for $\mathbf{4}$ appear at 1.70 and $1.92 \mathrm{ppm}$, respectively). The ${ }^{31} \mathrm{P}\left\{{ }^{1} \mathrm{H}\right\} \mathrm{NMR}$ spectra of $\mathbf{3}$ and $\mathbf{4}$ exhibit resonances at -8.34 and 11.01 ppm; nearly 4.7 and 4.3 ppm downfield shift compared to the corresponding parent molecules $\mathbf{1}$ and $\mathbf{2}$. The Li resonance in ${ }^{7} \mathrm{Li}$ NMR of $\mathbf{3}$ and $\mathbf{4}$ are observed, respectively, at 1.18 and $0.92 \mathrm{ppm}$. Crystals of $\mathbf{3}$ and 4 suitable for single crystal $\mathrm{X}$-ray analysis were grown from toluene and $\mathrm{C}_{6} \mathrm{D}_{6}$, respectively. Compounds 3 and 4 crystallize in monoclinic systems $\left(P 2_{1} / n\right.$ and $\left.P 2_{1} / c\right)$ (table 1 and figures 3 and 4).

Both 3 and $\mathbf{4}$ in the solid state exist as discrete $\mathrm{N}_{2}$ PLi nearly planar four-membered rings with Li having four as coordination number including two $\mathrm{N}$ atoms from a molecule of tmeda. The uniform $\mathrm{P}-\mathrm{N}$ distances in compounds 3 and 4 (1.574(1)-1.594(2) $\AA$ ) within the $\mathrm{N}_{2} \mathrm{PLi}$ heterocycle indicate that the anionic charge is delocalized over the $\mathrm{N}-\mathrm{P}-\mathrm{N}$ framework. The $\mathrm{N}_{2} \mathrm{PLi}$ ring in complexes $\mathbf{3}$ and $\mathbf{4}$ is nearly planar and the two endocyclic angles subtended at the lithium atom, being bite angles are acute 75.18(1) and $84.83(1)^{\circ}$ for $\mathrm{N}(1)-\mathrm{Li}(1)-\mathrm{N}(2)$ and $\mathrm{N}(3)-\mathrm{Li}(1)-\mathrm{N}(4)$, respectively, for compound 3 . The corresponding values for $\mathbf{4}$ are $73.95(2)^{\circ}$ and $83.63(1)^{\circ}$. The two aryl substituents on the terminal $\mathrm{N}$ atoms are mutually transoid. The four $\mathrm{Li}-\mathrm{N}$ bond lengths fall in the range from $2.010(1)$ to $2.196(1) \AA$, consistent with values
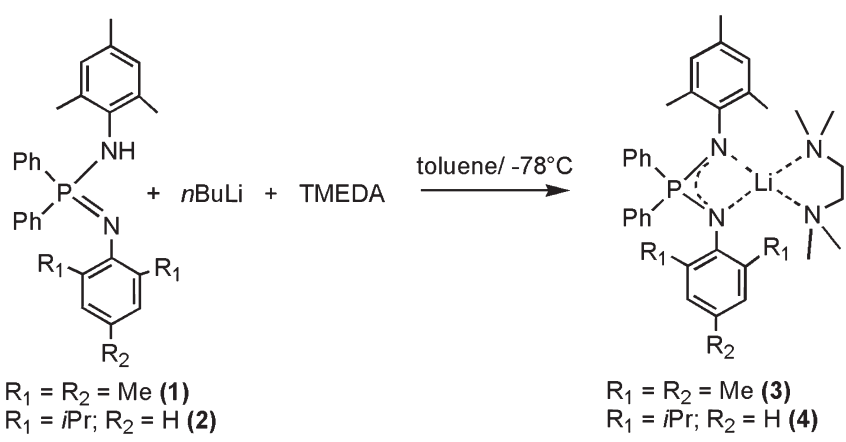

$R_{1}=R_{2}=M e(3)$ $\mathrm{R}_{1}=\mathrm{iPr} ; \mathrm{R}_{2}=\mathrm{H}(4)$

Scheme 2. Preparation of the tmeda adducts of lithium derivatives $\mathbf{3}$ and $\mathbf{4}$. 


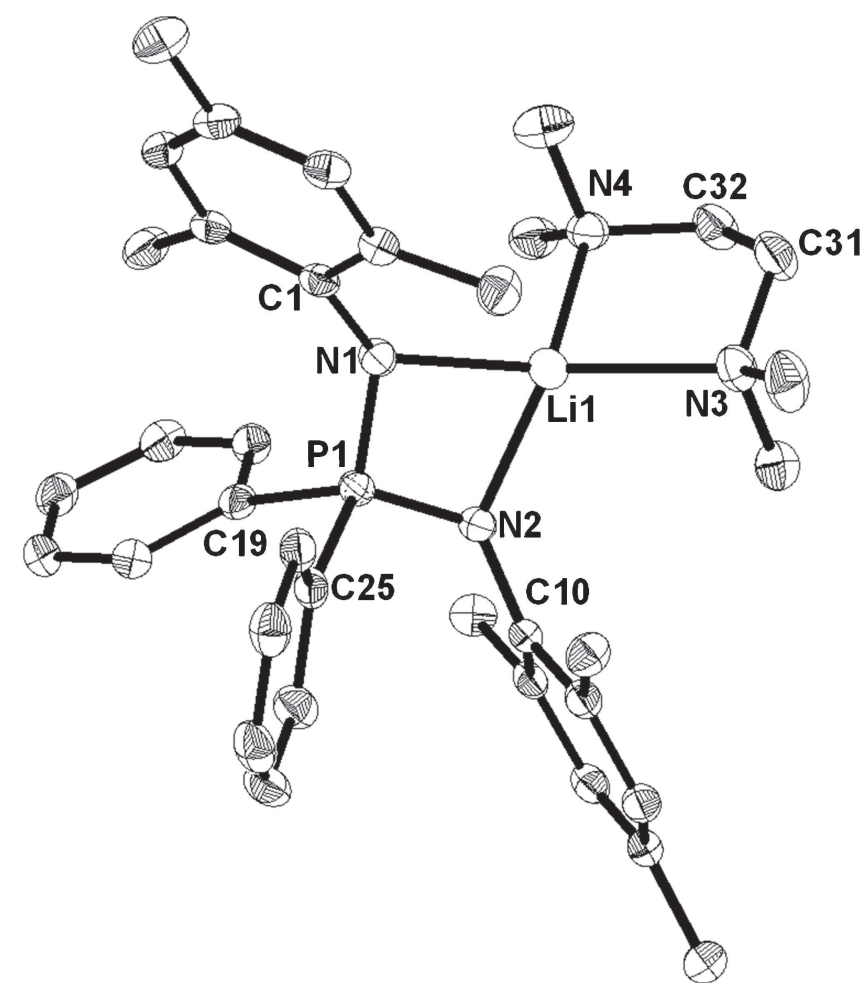

Figure 3. Single crystal X-ray structure of 3. All hydrogen atoms are omitted for clarity. Thermal ellipsoids are drawn at $50 \%$ probability. Selected bond lengths $(\AA)$ and bond angles $\left(^{\circ}\right)$ : $\mathrm{Li}(1)-\mathrm{N}(1) 2.085(3), \operatorname{Li}(1)-\mathrm{N}(2)$ 2.010(3), Li(1)$\mathrm{N}(3)$ 2.196(3), Li(1)-N(4) 2.104(3), P(1)-N(1) 1.591(1), $\mathrm{P}(1)-\mathrm{N}(2) 1.591(1) ; \mathrm{N}(1)-\mathrm{P}(1)-\mathrm{N}(2)$ 103.47(1), N(1)-Li(1)$\mathrm{N}(2) 75.18(2), \mathrm{N}(3)-\mathrm{Li}(1)-\mathrm{N}(4)$ 84.83(1), N(1)-Li(1)-N(4) 111.33(1), N(2)-Li(1)-N(4) 128.37(2).

observed in $\mathrm{Li}(\mathrm{Py})_{2}\left[\mathrm{Ph}_{2} \mathrm{P}\left(\mathrm{NSiMe}_{3}\right)_{2}\right]$ (2.043-2.123 $\AA),{ }^{19} \mathrm{Li}(\mathrm{THF})_{2}\left[\mathrm{Ph}_{2} \mathrm{P}\left(\mathrm{N} \mathrm{SiMe}_{3}\right)_{2}\right](2.04-2.08 \AA) .{ }^{17 \mathrm{a}}$

The moderately air-sensitive bis-iminophosphonamide complexes of $\mathrm{Co}$ (II) (5) and $\mathrm{Cu}$ (II) (6) with iminophosphonamine $\mathbf{1}$ were prepared as outlined in scheme 3 . The in situ generated metal silylamides were reacted with the iminophosphonamine $\mathbf{1}$.

Irrespective of the ligand and the metal silylamide stoichiometric ratio, the product formed is always the bis-homoleptic complex of iminophosphonamide and the yield can be improved by using 2:1 stoichiometry of ligand and metal silylamide. This probably is an indication that the intermediate heteroleptic monophosphonamides of $\mathbf{5}$ and $\mathbf{6}$ are more labile towards the formation of bis-homoleptic-chelate of these metal ions.

The molecular ion in the EI mass spectrum of $\mathbf{5}$ is revealed at $m / z 961$ and that of $\mathbf{6}$ at $m / z$ 967, whereas the base peak for both $\mathbf{5}$ and $\mathbf{6}$ is observed at $m / z 453$ corresponding to the free ligand. Complex $\mathbf{5}$ crystallizes in triclinic system with space group $P-1$ and two molecules of $\mathbf{5}$ are present in the unit cell (table 1). The X-ray structure of $\mathbf{5}$ shows that cobalt atom is

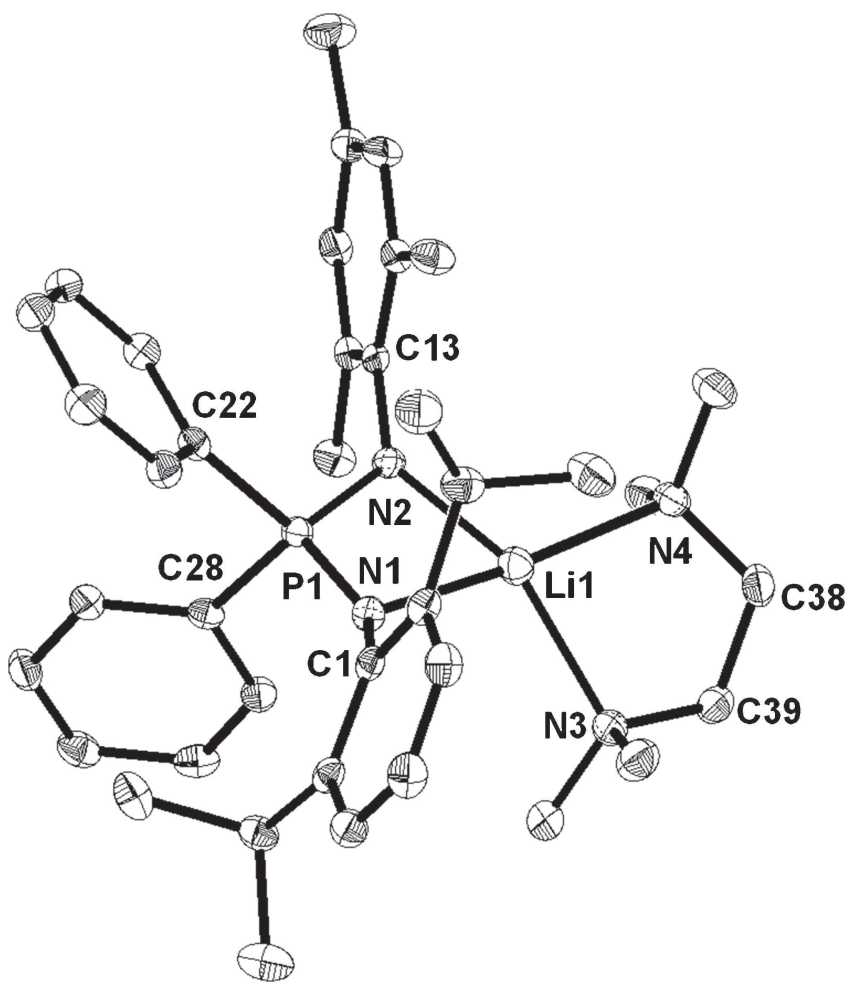

Figure 4. Molecular structure of 4. All hydrogen atoms and disordered toluene molecule in the crystal lattice are deleted for clarity. Thermal ellipsoids are drawn at $50 \%$ probability. Selected bond lengths $(\AA)$ and bond angles $\left({ }^{\circ}\right)$ : $\mathrm{Li}(1)-\mathrm{N}(1) 2.107(3), \mathrm{Li}(1)-\mathrm{N}(2)$ 2.063(3), Li(1)$\mathrm{N}(3) 2.199(3), \mathrm{Li}(1)-\mathrm{N}(4) 2.139(3), \mathrm{P}(1)-\mathrm{N}(1)$ 1.574(1), $\mathrm{P}(1)-\mathrm{N}(2)$ 1.594(1); N(1)-P(1)-N(2) 104.73(1), N(1)-Li(1)$\mathrm{N}(2) \quad 73.95(2), \quad \mathrm{N}(1)-\mathrm{Li}(1)-\mathrm{N}(3)$ 100.70(1), N(1)-Li(1)$\mathrm{N}(4)$ 169.95(1), N(2)-Li(1)-N(3) 151.78(1), N(2)-Li(1)$\mathrm{N}(4)$ 106.45(1), N(3)-Li(1)-N(4) 83.63(1).

encapsulated in a four-membered non-planar $\mathrm{CoN}_{2} \mathrm{P}$ metallacycle as $\eta^{2}$-chelates with two ligand units (figure 5). The bond angles at cobalt range from $73.57(1)$ to $143.27(2)^{\circ}$ with considerable distortion from tetrahedral geometry and the mesityl groups on the ligand $\mathrm{N}$ atoms are placed in such a way that the steric congestion between them is minimized. The Co atom deviates from the plane of $\mathrm{N}_{2} \mathrm{P}$ moiety by 0.089 and $0.108 \AA$ for $\mathrm{P}(1)$ and $\mathrm{P}(2)$ containing ligand scaffolds, respectively, and the dihedral angle between the two planes is ca. $88.01^{\circ}$. The four $\mathrm{Co}-$ $\mathrm{N}$ bond lengths lie within 2.015(1)-2.045(1) $\AA$, consistent with the only structurally characterized iminophosphonamides of $\mathrm{Co}(\mathrm{II}) \quad\left[\mathrm{Co}\left\{\mathrm{Ph}_{2} \mathrm{P}\left(\mathrm{N}-p-t \mathrm{BuC}_{6} \mathrm{H}_{4}\right)_{2}\right\}_{2}\right]$ (1.999-2.025 $\AA$ ). ${ }^{34}$ The anionic charges of both the iminophosphonamide ligands are delocalized over their respective $\mathrm{N}-\mathrm{P}-\mathrm{N}$ backbones (with four similar $\mathrm{P}-\mathrm{N}$ bond lengths $\mathrm{P}(1)-\mathrm{N}(1)$ 1.614(2), $\mathrm{P}(1)-\mathrm{N}(2)$ 1.609(3), $\mathrm{P}(2)-\mathrm{N}(3)$ 1.607(3), P(2)-N(4) 1.605(2) $\AA$ ). The bite angles of $\mathrm{N}(1)-\mathrm{Co}(1)-\mathrm{N}(2)$ and $\mathrm{N}(3)-\mathrm{Co}(1)-\mathrm{N}(4)$ are $73.83(1)$ and $73.57(1)^{\circ}$, respectively. 
<smiles>CN([Mg][Mg])c1ccccc1</smiles>

(1)

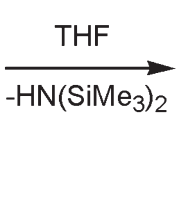

$$
\begin{aligned}
& \mathrm{Ar}=2,4,6-\mathrm{Me}_{3} \mathrm{C}_{6} \mathrm{H}_{2} \\
& \mathrm{M}=\mathrm{Co}(5), \mathrm{Cu}(6)
\end{aligned}
$$

Scheme 3. Preparation of bis-iminophosphonamide of cobalt(II) 5 and copper(II) 6.

Complex 6 crystallizes in triclinic system with $P-1$ space group and the asymmetric unit contains half a molecule of complex 6 and half a molecule of disordered toluene (table 1). The copper atom in $\mathbf{6}$ resides in a square planar environment formed by two $\eta^{2}$-chelates from two iminophosphonamide ions, the mesityl substituents on the ligand $\mathrm{N}$ atoms are oriented in such a way to minimize the steric crowding between them (figure 6). The copper atom lies exactly in the plane of four nitrogen atoms and $\mathrm{P}$ atoms are $0.097 \AA$ away from this plane. As in complex 5, four similar N-P bond lengths have also been observed for $6[\mathrm{P}(1)-\mathrm{N}(1)$ 1.608(1) and $\mathrm{P}(1)-\mathrm{N}(2)$

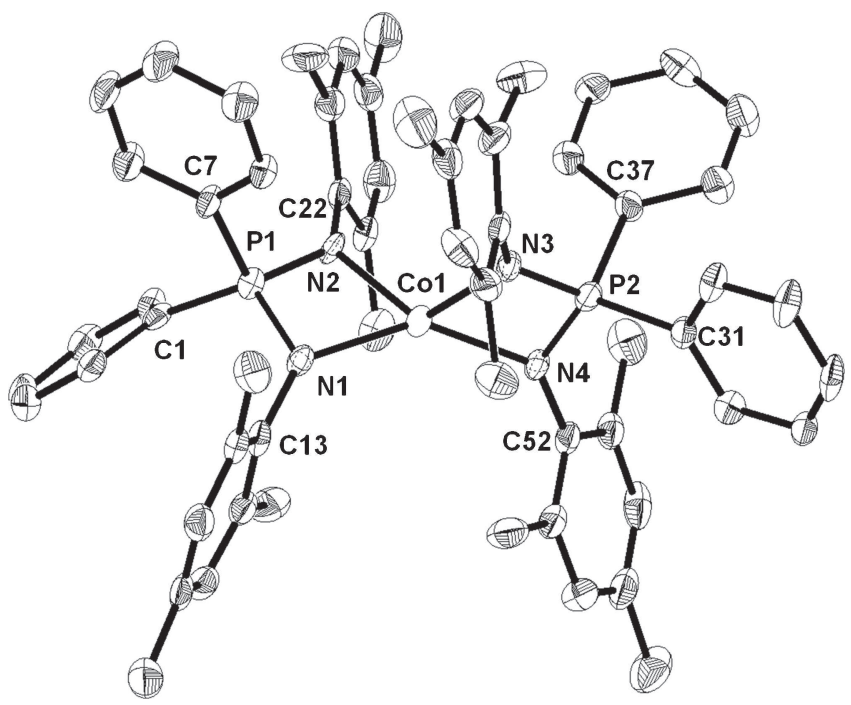

Figure 5. Molecular structure bis-iminophosphonamide of cobalt(II) 5. All hydrogen atoms are omitted for clarity. Thermal ellipsoids are drawn at $50 \%$ probability. Selected bond lengths $(\AA)$ and bond angles $\left({ }^{\circ}\right)$ : $\mathrm{Co}(1)-\mathrm{N}(1) \quad 2.015(1), \quad \mathrm{Co}(1)-\mathrm{N}(2) \quad 2.038(2), \quad \mathrm{Co}(1)-\mathrm{N}(3)$ 2.017(1), Co(1)-N(4) 2.045(1), P(1)-N(1) 1.614(1), P(1)$\mathrm{N}(2) 1.609(3), \mathrm{P}(2)-\mathrm{N}(3)$ 1.607(1), $\mathrm{P}(2)-\mathrm{N}(4)$ 1.605(2); $\mathrm{N}(1)-\mathrm{Co}(1)-\mathrm{N}(2) \quad 73.83(1), \quad \mathrm{N}(3)-\mathrm{Co}(1)-\mathrm{N}(4) \quad 73.57(2)$, $\mathrm{N}(2)-\mathrm{Co}(1)-\mathrm{N}(3)$ 121.14(2), N(1)-Co(1)-N(3) 125.29(1), $\mathrm{N}(2)-\mathrm{Co}(1)-\mathrm{N}(4)$ 127.04(2), N(1)-Co(1)-N(4) 143.27(1), $\mathrm{N}(1)-\mathrm{P}(1)-\mathrm{N}(2)$ 98.11(1), N(3)-P(2)-N(4) 98.45(1).
1.621(1) $\mathrm{A}$. The $\mathrm{Cu}(1)-\mathrm{N}(2)$ distance $(1.988(1) \AA)$ is slightly shorter than that of $\mathrm{Cu}(1)-\mathrm{N}(1)(2.018(1) \AA)$. The bite angles of $\mathrm{N}(1)-\mathrm{Cu}(1)-\mathrm{N}(2)$ and $\mathrm{N}(1)-\mathrm{P}(1)-$ $\mathrm{N}(2)$ are $75.74(1)$ and $99.20(2)^{\circ}$, respectively.

A nickel(II)-bis-iminophosphonamide, $\left[\mathrm{PPh}_{2}\{\mathrm{~N}(2,4\right.$, 6- $\left.\left.\left.\mathrm{Me}_{3} \mathrm{C}_{6} \mathrm{H}_{2}\right)\right\}_{2}\right] \mathrm{Ni}$ (7) was prepared in a reaction of $\mathrm{Li}\left[\left(2,4,6-\mathrm{Me}_{3} \mathrm{C}_{6} \mathrm{H}_{2}\right) \mathrm{NP}\left(\mathrm{Ph}_{2}\right)=\mathrm{N}\left(\mathrm{C}_{6} \mathrm{H}_{2}-2,4,6-\mathrm{Me}_{3}\right)\right]$ with half equivalent of $\left(\mathrm{PPh}_{3}\right)_{2} \mathrm{NiCl}_{2}$ in toluene (scheme 4)

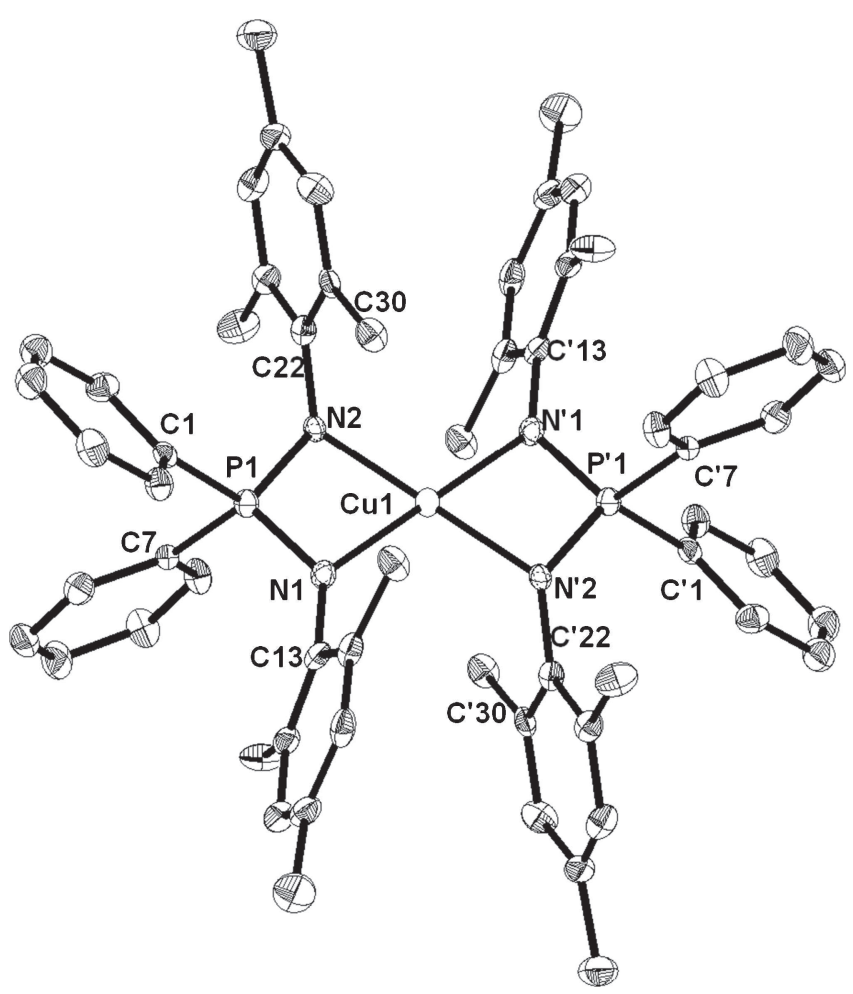

Figure 6. Molecular structure of bis-iminophosphonamide of copper(II) 6. Disordered toluene molecule and all hydrogen atoms are omitted for clarity. Thermal ellipsoids are drawn at $50 \%$ probability. Selected bond lengths $(\AA)$ and bond angles $\left({ }^{\circ}\right)$ : $\mathrm{Cu}(1)-\mathrm{N}(1) 2.018(1), \mathrm{Cu}(1)-$ $\mathrm{N}(2)$ 1.988(2), $\mathrm{P}(1)-\mathrm{N}(1)$ 1.608(1), $\mathrm{P}(1)-\mathrm{N}(2)$ 1.621(1); $\mathrm{N}(1)-\mathrm{Cu}(1)-\mathrm{N}(2) 75.74(1), \mathrm{N}(1)-\mathrm{P}(1)-\mathrm{N}(2) 99.20(1), \mathrm{N}(2)-$ $\mathrm{Cu}(1)-\mathrm{N}^{\prime}(2)$ 179.99(2), N(1)-Cu(1)-N'(1) 179.99(2), N(2)$\mathrm{Cu}(1)-\mathrm{N}^{\prime}(1)$ 104.26(1), N(1)-Cu(1)-N'(2) 104.26(1). 
at $-30^{\circ} \mathrm{C}$. The initial green solution gradually turned to reddish brown, and $\mathbf{7}$ was crystallized from the same solvent as reddish-brown crystals (76\% isolated yield). The ${ }^{1} \mathrm{H}$ NMR spectrum of 7 reveals one singlet that appears at 1.67 corresponding to $p-\mathrm{Me}$ of the mesityl group; this is up-field shifted compared to the free ligand $(2.05 \mathrm{ppm})$. The $o$-Me groups in 7 become magnetically non-equivalent and appear as overlapped singlets between 2.03 and $2.09 \mathrm{ppm}$; these $\mathrm{H}$ atoms in 7 are downfield shifted compared to the free ligand (1.92 ppm). This magnetic non-equivalency can be attributed to weak agostic interaction of these remote $o$-Me hydrogen atoms that interact with $\mathrm{Ni}(\mathrm{II})$ to occupy the vacant axial sites of the octahedron geometry in 7 (figure 7).

Other $6 o$-Me groups that are oriented away from $\mathrm{Ni}$ (II) do not participate in any such interaction. The same was also observed in the solid state structure of 7 , vide infra. The $m-\mathrm{H}$ of mesityl group appears as two singlets at 6.48 and $6.61 \mathrm{ppm}$. Other aromatic hydrogen atoms of $\mathbf{7}$ appear as complex multiplets between 7.28-7.32, 7.35-7.39 and 7.76-7.81 ppm. The ${ }^{31} \mathrm{P}\left\{{ }^{1} \mathrm{H}\right\}$ NMR spectrum for this compound shows a single resonance at $-16.83 \mathrm{ppm}$, an up-field shift compared to $\mathbf{1}$. The molecular ion peak in the EI mass spectrum of 7 was observed at $m / z$ 962. Complex 7 crystallizes in triclinic system with $P-1$ space group (table 1) with half a molecule of 7 and half a molecule of disordered toluene in an asymmetric unit. As shown in figure 7, complex 7 is structurally similar to complex $\mathbf{6}$ with four nitrogen atoms from two ligand units in an essentially square planar arrangement around nickel. However, unlike $\mathbf{6}$ the P atoms in 7 also lie in this plane thus making the four-membered metallacycles co-planar. The mesityl groups on the nitrogen atoms occupy planes oriented at 127.99(2) and $129.72(2)^{\circ}$ to this plane and the average $\mathrm{Ni}-\mathrm{N}$ bond distance is $1.943 \AA$. The monoanionic charge is delocalized over the NPN backbone, $[\mathrm{P}(1)-\mathrm{N}(1)$ $1.610(2)$ and $\mathrm{P}(1)-\mathrm{N}(2) 1.599(2) \AA]$. The chelate rings have acute $\mathrm{N}(1)-\mathrm{Ni}(1)-\mathrm{N}(2)$ angle as 76.44(2) and

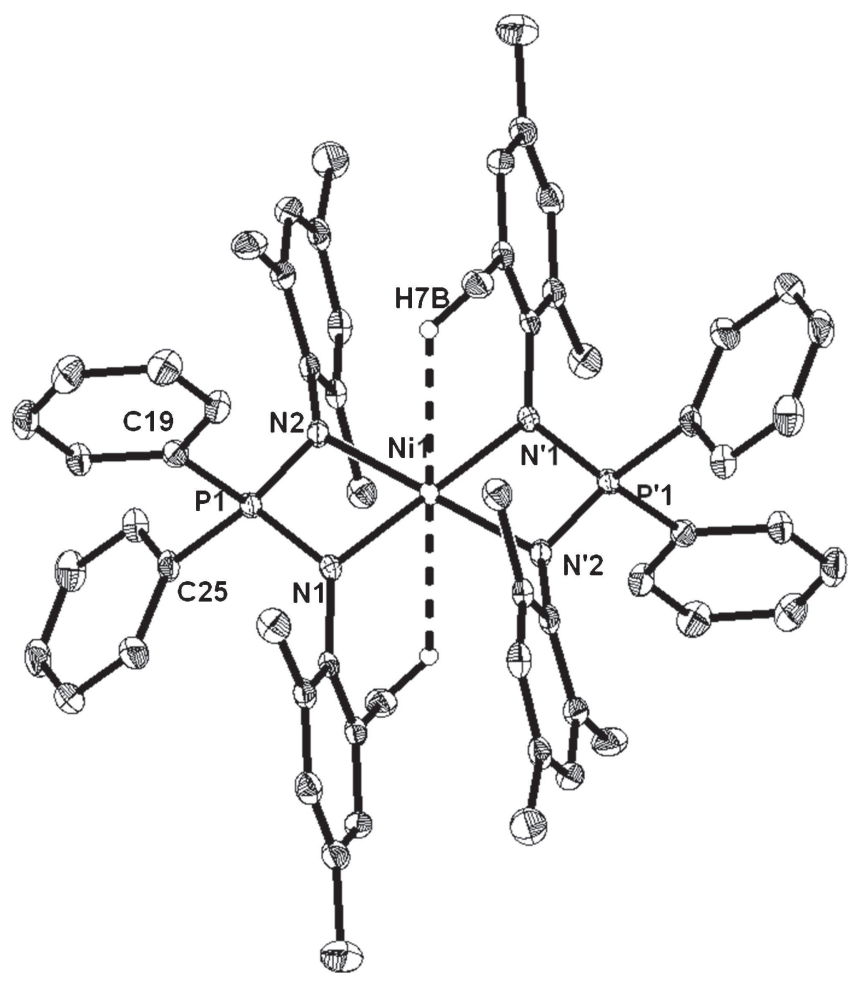

Figure 7. Single crystal X-ray structure of 7. Disordered toluene molecule and all hydrogen atoms, except those involved in $\mathrm{Ni} \cdot \mathrm{H}$ interaction, are omitted for clarity. Thermal ellipsoids are drawn at 50\% probability. Selected bond lengths $(\AA)$ and bond angles $\left({ }^{\circ}\right)$ : $\mathrm{Ni}(1)-\mathrm{N}(1)$ 1.942(2), Ni(1)$\mathrm{N}(2)$ 1.944(2)，P(1)-N(1) 1.610(2)，P(1)-N(2) 1.599(2), $\mathrm{P}(1)-\mathrm{C}(25)$ 1.814(1), $\mathrm{P}(1)-\mathrm{C}(19) \quad 1.815(2) ; \mathrm{N}(1)-\mathrm{P}(1)-$ $\mathrm{N}(2) \quad 97.06(2), \quad \mathrm{N}(1)-\mathrm{Ni}(1)-\mathrm{N}(2) \quad 76.44(2), \mathrm{N}^{\prime}(1)-\mathrm{Ni}(1)-$ $\mathrm{N}(2) 103.56(2), \mathrm{N}(1)-\mathrm{Ni}(1)-\mathrm{N}^{\prime}(1) 180.00(2), \mathrm{N}(2)-\mathrm{Ni}(1)-$ N'(2) 180.00(2).

$\mathrm{N}(1)-\mathrm{P}(1)-\mathrm{N}(2)$ angle as $97.06(2)^{\circ}$ consistent with values previously reported for $\left[\mathrm{Ni}\left\{\mathrm{Ph}_{2} \mathrm{P}\left(\mathrm{NSiMe}_{3}\right)_{2}\right\}_{2}\right]{ }^{39}$ The very weak $\mathrm{Ni} \cdots \mathrm{H}_{\delta}$ agostic interaction of $\mathrm{Ni}(1)$ and $\mathrm{H}(7 \mathrm{~B})$ measures 2.673(1) $\AA$ and is shorter than that seen in $\left[\left\{\mathrm{PPh}_{2}\left(\mathrm{NSiMe}_{3}\right)_{2}\right\} \mathrm{Ni} \mathrm{Ph}\left(\mathrm{PPh}_{3}\right)\right]\left(\mathrm{Ni} \cdots \mathrm{H}_{\gamma} 2.81\right.$ $\AA) .{ }^{37}$ This interaction is probably responsible for magnetic non-equivalence of $o$-Me groups that interact with $\mathrm{Ni}$ to that of those not involved in this interaction (vide supra).

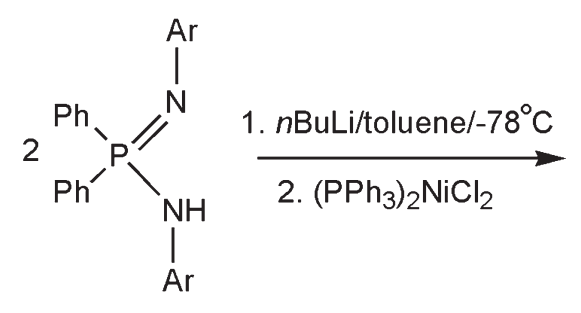

(1)

$\mathrm{Ar}=2,4,6-\mathrm{Me}_{3} \mathrm{C}_{6} \mathrm{H}_{2}$

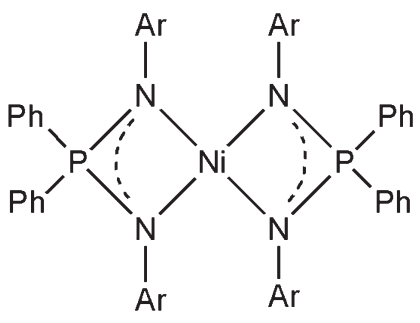

(7)

Scheme 4. Synthesis of bis-iminophosphonamide of nickel(II) 7. 


\section{Conclusions}

We have synthesized and structurally characterized iminophosphonamine ligands containing sterically bulky substituents. The ligands can be easily deprotonated by alkyllithiums or transition metal silylamides. The lithium iminophosphonamides have been isolated as tmeda adducts and a lithium iminophosphonamide has been used to prepare bis-homoleptic complex of $\mathrm{Ni}(\mathrm{II})$. The neutral iminophosphonamines is also a suitable precursor to prepare metal complexes, for example, bis-homoleptic complexes of $\mathrm{Co}$ (II) and $\mathrm{Cu}$ (II) have been prepared and structurally characterized by using bis-silylamide of the respective metals. It is expected that the same synthetic strategy can be adopted for the synthesis of main group metal complexes as well. Such studies are currently underway in our laboratory.

\section{Supplementary Information}

CCDC 928556-928562 contains the supplementary crystallographic data for compounds 1-7. These data can be obtained free of charge from The Cambridge Crystallographic Data Centre via www.ccdc.cam.ac.uk/ data_request/cif.

\section{Acknowledgements}

We acknowledge the financial support, Grant No. SR/FT/CS-009/2009, from the Department of Science and Technology (DST), India. The authors thank Indian Institute of Science Education and Research Mohali (IISER) Mohali for NMR, X-ray facility and all other infrastructural support.

\section{References}

1. (a) Fedorchuk C, Copsey M and Chivers T 2007 Coord. Chem. Rev. 251 897; (b) Chivers T, Eisler D J, Fedorchuk C, Schatte G, Tuononen H M and Boere R T 2006 Inorg. Chem. 45 2119; (c) Chivers T, Fedorchuk C, Schatte G and Parvez M 2003 Inorg. Chem. 42 2084; (d) Chivers T, Fedorchuk C and Parvez M 2004 Inorg. Chem. 43 2643; (e) Harder S 2010 Dalton Trans. 39 6677

2. Edelmann F T 1994 Coord. Chem. Rev. 137403

3. Hauber S-O, Lissner F, Deacon G B and Niemeyer M 2005 Angew. Chem. Int. Ed. 445871

4. Horner M, Oliveira G M, Naue J A, Daniels J and Beck J 2006 J. Organomet. Chem. 6911051

5. Nimitsiriwat $\mathrm{N}$, Gibson $\mathrm{V}$ C, Marshal E L, Takolpuckdee P, Tomov A K, White A J P, Williams D J, Elsegood M R J and Dale S H 2007 Inorg. Chem. 469988
6. Hauber S-O and Niemeyer M 2005 Inorg. Chem. 44 8644

7. Alexander S G, Cole M L, Forsyth C M, Furfari S K and Kontas K 2009 Dalton Trans. 2326

8. Lee H S and Niemeyer M 2011 Inorg. Chim. Acta 374 163

9. Roy C G, Butcher R J and Bhattacharya S $2008 \mathrm{~J}$. Organomet. Chem. 6933923

10. Meinholz M M, Pandey S K, Deuerlein S M and Stalke D 2011 Dalton Trans. 401662

11. Pauer F, Rocha J and Stalke D 1991 J. Chem. Soc. Chem. Commun. 1477

12. Fleischer R, Walfort B, Gbureck A, Scholz P, Kiefer W and Stalke D 1998 Chem. Eur. J. 42266

13. Pauer F and Stalke D 1991 J. Organomet. Chem. 418 127

14. Edelmann F T, Knosel F, Pauer F, Stalke D and Bauer W 1992 J. Organomet. Chem. 438, 1

15. Steiner A, Zacchini S and Richards P I 2002 Coord. Chem. Rev. 227193

16. Witt M and Roesky H W 1994 Chem. Rev. 941163

17. (a) Steiner A and Stalke D 1993 Inorg. Chem. 32 1977; (b) Edwards A J and Wenger E 2002 Aust. J. Chem. 55 249

18. Schultz M, Straub B F and Hofmann P 2002 Acta Crystallogr. Sec. C $\mathbf{5 8} \mathrm{m} 256$

19. Müller E, Müller J, Schimidt H-G, Noltemeyer M and Edelmann F T 1996 Phosphorus Sulfur Silicon Relat. Elem. 119121

20. Wingerter S, Pfeiffer M, Murso A, Lustig C, Stey T, Chandrasekhar V and Stalke D $2001 \mathrm{~J}$. Am. Chem. Soc. 1231381

21. Ahmed S A, Hill M S, Hitchcock P B, Mansell S M and John O S 2007 Organometallics 26538

22. Kocher N, Leusser D, Murso A and Stalke D 2004 Chem. Eur. J. 103622

23. Kornev A N, Sushev V V, Panova Y S, Belina N V, Lukoyanova O V, Fukin G K, Ketkov S Y, Abakumov G A, Lonnecke P and Hawkins E H 2012 Inorg. Chem. 51874

24. Fleischer R and Stalke D 1997 Inorg. Chem. 362413

25. Nekoueishahraki B, Roesky H W, Schwab G, Stern D and Stalke D 2009 Inorg. Chem. 489174

26. Vollmerhaus R, Shao P, Taylor N J and Collins S 1999 Organometallics 182731

27. Vollmerhaus R, Tomaszewski R, Shao P, Taylor N J, Wiacek K J, Lewis S P, Al-Humydi A and Collins S 2005 Organometallics $\mathbf{2 4} 494$

28. Tomaszewski R, Vollmerhaus R, Al-Humydi A, Wang Q, Taylor N J and Collins S 2006 Can. J. Chem. 84214

29. Li S, Miao W, Tang T, Dong W, Zhang X and Cui D 2008 Organometallics 27718

30. Li S, Cui D, Li D and Hou Z 2009 Organometallics 28 4814

31. Peganova T A, Valyaeva A V, Kalsin A M, Petrovskii P V, Borissova A O, Lyssenko K A and Ustynyuk N A 2009 Organometallics 283021

32. (a) Stasch A 2012 Chem. Eur. J. 18, 15105; (b) Albahily K, Licciulli S, Gambarotta S, Korobkov I, Chevalier R, Schuhen K and Duchateau R 2011 Organometallics 30 3346

33. Prashanth B, Karanam M, Choudhury A R and Singh S 2012 Polyhedron 47112 
34. Fedotova Y V, Kornev A N, Sushev V V, Kursky Y A, Mushtina T G, Makarenko N P, Fukin G K, Abakumov G A, Zakharov L N and Rheingold A L 2004 J. Organomet. Chem. 6893060

35. Straub B F, Gruber I, Rominger F and Hofmann P 2003 J. Organomet. Chem. 684124

36. Shishkov I V, Rominger F and Hofmann P 2009 Organometallics $\mathbf{2 8} 1049$

37. Stapleton R L, Chai J, Taylor N J and Collins S 2006 Organometallics 252514

38. Fenske D, Maczek B and Maczek K 1997 Z. Anorg. Allg. Chem. 6231113

39. Boese R, Düppmann M, Kuchen W and Peters W 1998 Z. Anorg. Allg. Chem. 624837
40. Greenwood B P, Forman S I, Rowe G T, Chen C-H, Foxman B M and Thomas C M 2009 Inorg. Chem. 48 6251

41. Stasch A 2012 Angew. Chem. Int. Ed. 511930

42. Murata S, Abe S and Tomioka H 1997 J. Org. Chem. 62 3055

43. Liu Q and Tor Y 2003 Org. Lett. 142571

44. Bürger H and Wannagat U 1963 Monatsh. Chem. 94 1007

45. Murray B D and Power P P 1984 Inorg. Chem. 234584

46. Andersen R A, Feegri K, Green J C, Haaland A, Lappert M F, Leung W-P and Rypdal K 1998 Inorg. Chem. 27 1782

47. Sheldrick G M 2008 Acta Crystallogr. Sec. A 64112 